\title{
Effect of Genotype on Growth Traits Characteristics of Two Commercial Broiler Chickens in a Derived Savannah Zone of Nigeria
}

\author{
Ige, A.O*., Rafiu, B.R., Mudasiru, I.T \\ Department of Animal Nutrition and Biotechnology, Ladoke Akintola University of Technology, \\ Ogbomoso Oyo State Nigeria. \\ *aoige@lautech.edu.ng (Author for Correspondence)
}

\begin{abstract}
This study is aimed to investigate the effect of genotype on growth traits characteristics of Hubbard and Arbor acre commercial broiler chickens treated under the same dietary and environmental conditions. A total of one hundred (100) broiler chicks were used for the experiment consisted of Hubbard and Arbor Acre strain of broiler chicken. Data were collected from 1 week to 8 week of age on body weight using sensitive weighing balance and body measurements (shank length, wing length, thigh length, chest circumference, keel length and body length) were measured from each bird using a tailor's tape. All data were subjected to General Linear Model (GLM) using SAS 2000 software package. The correlations between body weight and linear body measurements were determined using Pearson's correlation coefficient ( $r$ ). The result indicated that body weight, body length, chest circumference, keel length and wing length were significant $(p<0.05)$ with male Hubbard broiler strain had consistence superiority inbody weight $654.00 \pm 22.68 \mathrm{~g}, 862.00 \pm 26.63 g$, $1133.83 \pm 68.90 \mathrm{~g}, 1438.50 \pm 61.82 \mathrm{~g}$ and $1969.70 \pm 156.93 \mathrm{~g}$ at $4,5,6,7$ and 8 week of age respectively. Also female Hubbard strain attained the body weight of $112.25 \pm 7.25 \mathrm{~g}, 218.67 \pm 17.52 \mathrm{~g}, 449.33 \pm 75.93 \mathrm{~g}, 627.67 \pm 65.46 \mathrm{~g}$, $846.33 \pm 27.47 \mathrm{~g}, 1062.00 \pm 72.10 \mathrm{~g}$ and $1326.00 \pm 168.35 \mathrm{~g}$ at $1,2,4,5,6,7$ and 8 week of age respectively. Hubbard broiler strain showed superiority in growth assessment of body weight and linear body measurements in the 1 to 8 weeks study period.The result also showed that there were very high significant $(p<0.0001)$ positive correlation between body weight and body measurements of Hubbard strain and Arbor acre strain of broiler chicken. The correlation between body weight and body measurements in female Arbor acre ranged from 0.86 to 0.98 while male Arbor acre ranged from 0.94 to 0.98. The correlation between body weight and body measurements in female Hubbard ranged from 0.72 to 0.92 while male Hubbard ranged from 0.72 to 0.93 . From this study, Hubbard broiler strain has been seen to exhibit significant higher body weight and body measurements over Abor acre strain of broiler. The results of this study also suggest that there are high positive correlations between body weight and linear body measurements.
\end{abstract}

Keywords: Strain, body measurement, correlation and derived savannah.

\section{INTRODUCTION}

The poultry industry has made tremendous adjustments to meet the increasing demand for inexpensive and safe supply of meat and eggs. Over the past three decades, the poultry sector has been growing at more than 5 percent per annum (compared to 3 percent for pig meat and 1.5 percent for bovine meat) and its share in world meat production increased from 15 percent three decades ago to 30 percent currently (FAO, 2006a). Poultry production is considered to be one of the most popular options in Nigeria in reducing the incidence of malnutrition particularly protein deficiency in the diets of populace (Obasoyaet al., 2005). This sector ranks among the fastest growing segment of the livestock industry in Nigeria just like in most developing countries all over the world (Oluyemi and Roberts 2000; Cahaner 2008). Chicken meat is unique in that its price is comparatively low, easy to partition into smaller parts and no religious restriction against its consumption (Jaturasitha, 2004). Broiler has a great demand as compared to other meat, because of the spiritual beliefs as well as religious constraints in the case of pork and beef (Jabbar, 2004).Regardless of genetic improvements performed by breeders, broiler hybrids still differ with regard to their efficiency due to the specific selection practices (Emmerson, 1997).Furthermore, Ajayi and Ejiofor (2009) reported significant genotype $\mathrm{x}$ sex effect on live weight and body linear measurements in broiler breeds. In local fowls, Igeet al. (2007) reported positive phenotypic correlation between body weight and linear 
measurements, while Razukiet al. (2011) reported significant strain differences in body weight at different ages among breeds of broiler chickens.

Ige (2013) reported that high, positive and significant correlation coefficient between body weight and linear body measurement indicates that easily measured body parts can be used as criteria for selection of body weight in crossbred Fulani ecotype. Therefore, the present investigation aimed at determining the effect of genotype on growth traitscharacteristics of Hubbard and Arbor acre commercial broiler chickens in a derived savannah zone of Nigeria.

\section{Material StUdied}

\subsection{Area Description}

The experiment was conducted at the poultry unit of teaching and research farm, LadokeAkintola University of Technology, Ogbomoso, Oyo State, Nigeria.The study area is located between latitudes $8^{\circ} 07^{\prime}$ and $8^{\circ} 12^{\prime} \mathrm{N}$ and longitudes $4^{\circ} 04^{\prime}$ and $4^{\circ} 15^{\prime} \mathrm{E}$. The mean annual rainfall is $1247 \mathrm{~mm}$ with relative humidity of between 75 and $95 \%$. The location is situated at about $500 \mathrm{~mm}$ above the sea level with a mean annual temperature of $26.2^{\circ} \mathrm{C}$ (Oguntoyinbo, 1978).

\subsection{Experimental Birds}

A total of 100day old chicks made up of 50 chicks each of Arbor Acre and Hubbard commercial strains of broilers were used for the study. The chicks were procured from reputable Hatchery in Ibadan, Oyo State Nigeria. The chicks were vaccinated against marek disease, Newcastle disease and gumboro disease at the appropriate ages. Coccidiostat was administered in water periodically as a prophylactic measure. Each strain was housed in separate pens with three replicate for each strain consisted of both male and female.

\subsection{Housing and Feeding Management}

The brooding house was partitioned into pens in line with the design of the experiment. All the necessary repairs were made in the brooding house. The floors of the brooding pens were covered with wood shavings and they were kept dry throughout the experimental period by replacing the wet litter with new ones. Electricity and charcoal were used to heat up the brooding house. Shallow plastic feeders and chick founts were used to provide feed and water to the chicks respectively.Chicks were fed ad libitum on a broiler starter diet containing $23 \% \mathrm{CP}$ and $3000 \mathrm{Kcal} / \mathrm{kg} / \mathrm{ME}$ from day old to 4 weeks of age followed by a finisher diet containing $20 \% \mathrm{CP}$ and $2900 \mathrm{Kcal} / \mathrm{kg} / \mathrm{ME} 5$ to 8 weeks of age. Feed and water were made available to the birds all the time.

\subsection{Data Collection}

Data were collected on linear body measurementssuch as body length, chest circumference, keel length, shank length, thigh length and wing length. All these parameters were measured on weekly basis for eight (8) weeks, with tape rule in centimeter $(\mathrm{cm})$.

(a) Body weight gain $(g)$

This was calculated by subtracting final weight gain from initial weight gain. The initial body weight of the chicks was taken at day old using a sensitive balance. The body weight (BW) was measured in gram using sensitive scale ( $5 \mathrm{~kg}$, capacity). Subsequently, their body weights were taken every week for 8 weeks.

(b)Body Length

Body Length (BL) was measured as the linear distance between the nasal opening and the top of pygostlye when the neck is carefully stretched and the measuring tape positioned along the midline of the birds back.

\section{(c) Chest Circumference}

Chest Circumference (CC) was determined by winding a tape rule around the region of the breast.

(d) Shank Length

Shank length (SL) was measured as the distance from the foot pad to the hock joint.

(e) Thigh Length

Thigh length (TL) was measured from the tip of the tarsus to the ball joint.

(f) Keel length 
Effect of Genotype on Growth Traits Characteristics of Two Commercial Broiler Chickens in a Derived Savannah Zone of Nigeria

Keel length (KL) was measured from the cranial to the caudal terminals of the keel bone.

(g) Wing Length

Wing length (WL) was measured by stretching the wing and the measurement taken from humerous coracoids junctions to the tip of the digit.

(h) Statistical Analysis

All data generated were subjected to General Linear Model (GLM) using SAS (2003) and significant means were separated using Duncan Multiple Range Test of the same package.

\section{Results}

Table1. Estimate of body weight and body measurement as affected by strain and age of Hubbard and Arbor Acre male strains of broiler chickens

\begin{tabular}{|c|c|c|c|c|c|c|c|c|c|}
\hline \multicolumn{10}{|c|}{ Age } \\
\hline \multirow{3}{*}{$\begin{array}{l}\text { Traits } \\
\text { BDW } \\
\text { (g) }\end{array}$} & Strain & 1 & 2 & 3 & 4 & 5 & 6 & 7 & 8 \\
\hline & $\mathrm{H}$ & $124.50 \pm 8.00$ & $\begin{array}{l}236.33 \pm 11.2 \\
8\end{array}$ & $432.00 \pm 23.71$ & $\begin{array}{l}654.00 \pm 22 \\
.6^{\mathrm{a}}\end{array}$ & $\begin{array}{l}862.00 \pm 26 . \\
63^{\mathrm{a}}\end{array}$ & $\begin{array}{l}1133.83 \pm 68 . \\
90^{\mathrm{a}}\end{array}$ & $\begin{array}{l}1438.50 \pm 61 . \\
82^{\mathrm{a}}\end{array}$ & $\begin{array}{l}1969.70 \pm 156.9 \\
3^{\mathrm{a}}\end{array}$ \\
\hline & A & $114.20 \pm 4.39$ & $230.00 \pm 7.77$ & $402.00 \pm 45.00$ & $\begin{array}{l}508.67 \pm 7 . \\
04^{b}\end{array}$ & $\begin{array}{l}678.00 \pm 58 . \\
53^{\mathrm{b}}\end{array}$ & $\begin{array}{l}1084.67 \pm 42 . \\
21^{\mathrm{b}}\end{array}$ & $\begin{array}{l}1251.33 \pm 54 . \\
18^{\mathrm{b}}\end{array}$ & $1451.00 \pm 48.88^{b}$ \\
\hline \multirow[t]{2}{*}{$\begin{array}{l}\mathrm{BDL} \\
(\mathrm{cm})\end{array}$} & $\mathrm{H}$ & $9.00 \pm 0.00$ & $13.01 \pm 0.44^{\mathrm{a}}$ & $15.00 \pm 0.25^{\mathrm{a}}$ & $\begin{array}{l}16.38 \pm 0.2 \\
2\end{array}$ & $19.07 \pm 0.95$ & $19.82 \pm 1.02$ & $22.90 \pm 0.45^{\mathrm{a}}$ & $24.45 \pm 0.89^{\mathrm{a}}$ \\
\hline & A & $8.70 \pm 0.20$ & $11.43 \pm 0.28^{b}$ & $12.60 \pm 0.40^{\mathrm{b}}$ & $\begin{array}{l}15.63 \pm 0.7 \\
7\end{array}$ & $18.47 \pm 0.32$ & $19.37 \pm 0.26$ & $20.50 \pm 0.29^{b}$ & $21.87 \pm 0.19^{b}$ \\
\hline \multirow[t]{2}{*}{$\begin{array}{l}\mathrm{PC} \\
(\mathrm{cm})\end{array}$} & $\mathrm{H}$ & $10.18 \pm 0.08^{\mathrm{a}}$ & $12.98 \pm 0.54$ & $17.63 \pm 0.47^{\mathrm{a}}$ & $\begin{array}{l}17.93 \pm 0.6 \\
3\end{array}$ & $20.93 \pm 0.57^{\mathrm{a}}$ & $23.07 \pm 1.15$ & $24.83 \pm 1.72$ & $26.90 \pm 1.38$ \\
\hline & A & $7.93 \pm 0.57^{b}$ & $11.13 \pm 0.96$ & $15.55 \pm 0.55^{b}$ & $\begin{array}{l}16.98 \pm 0.4 \\
9\end{array}$ & $\begin{array}{l}18.90 \pm 0.41 \\
b\end{array}$ & $22.60 \pm 0.48$ & $24.13 \pm 0.94$ & $25.06 \pm 0.92$ \\
\hline \multirow{2}{*}{$\begin{array}{l}\mathrm{KL} \\
(\mathrm{cm})\end{array}$} & $\mathrm{H}$ & $3.88 \pm 0.28$ & $6.95 \pm 0.37^{\mathrm{a}}$ & $8.77 \pm 0.34$ & $9.93 \pm 0.55^{\circ}$ & $11.43 \pm 0.38^{\mathrm{a}}$ & $12.40 \pm 0.50^{\mathrm{a}}$ & $13.00 \pm 0.38^{\mathrm{a}}$ & $13.23 \pm 0.38^{\mathrm{a}}$ \\
\hline & $\mathrm{A}$ & $2.83 \pm 0.58$ & $4.77 \pm 0.37^{b}$ & $7.55 \pm 1.45$ & b $7.81 \pm 0.64$ & $8.20 \pm 0.49^{b}$ & $10.07 \pm 0.88^{b}$ & $10.60 \pm 1.43^{b}$ & $10.83 \pm 0.78^{b}$ \\
\hline \multirow{2}{*}{$\begin{array}{l}\mathrm{SHL} \\
(\mathrm{cm})\end{array}$} & $\mathrm{H}$ & $2.80 \pm 0.13$ & $3.83 \pm 0.42$ & $4.00 \pm 0.23$ & $4.58 \pm 0.25$ & $5.43 \pm 0.34$ & $5.63 \pm 0.15$ & $6.68 \pm 0.26$ & $7.58 \pm 0.10$ \\
\hline & $\mathrm{A}$ & $2.48 \pm 0.25$ & $3.37 \pm 0.03$ & $3.77 \pm 0.00$ & $4.50 \pm 0.35$ & $5.23 \pm 0.06$ & $5.60 \pm 0.20$ & $6.33 \pm 0.38$ & $6.53 \pm 0.69$ \\
\hline \multirow{2}{*}{$\begin{array}{l}\text { THL } \\
(\mathrm{cm})\end{array}$} & $\mathrm{H}$ & $4.75 \pm 0.25$ & $5.83 \pm 0.86$ & $6.50 \pm 0.46$ & $7.73 \pm 0.09$ & $9.43 \pm 0.39$ & $10.15 \pm 0.18$ & $11.35 \pm 0.48$ & $11.87 \pm 0.59$ \\
\hline & A & $3.80 \pm 0.35$ & $5.23 \pm 0.09$ & $6.20 \pm 0.70$ & $7.70 \pm 0.59$ & $9.13 \pm 0.38$ & $9.87 \pm 0.55$ & $11.33 \pm 0.73$ & $11.73 \pm 0.45$ \\
\hline \multirow[t]{2}{*}{$\begin{array}{l}\text { WL } \\
(\mathrm{cm})\end{array}$} & $\mathrm{H}$ & $11.00 \pm 0.00^{\mathrm{a}}$ & $13.65 \pm 0.53$ & $18.40 \pm 0.60^{\mathrm{a}}$ & $\begin{array}{l}20.90 \pm 1.1 \\
3\end{array}$ & $23.17 \pm 1.10$ & $25.77 \pm 0.77^{\mathrm{a}}$ & $27.47 \pm 0.73^{\mathrm{a}}$ & $28.85 \pm 2.23$ \\
\hline & A & $9.20 \pm 0.30^{b}$ & $12.90 \pm 0.95$ & $13.53 \pm 0.48^{b}$ & $\begin{array}{l}20.87 \pm 0.3 \\
3\end{array}$ & $22.40 \pm 1.09$ & $22.07 \pm 1.07^{b}$ & $25.85 \pm 0.57^{b}$ & $27.97 \pm 0.41$ \\
\hline
\end{tabular}

ab Mean values along the same column with different superscripts are significant at $p<0.05$ BDW $=$ Body Weight, $\mathrm{BDL}=$ Body Length, $\mathrm{CC}=$ Chest Circumference, $\mathrm{KL}=$ Keel Length, $\mathrm{SHL}=$ Shank Length, $\mathrm{THL}=$ Thigh Length, $\mathrm{WL}=$ Wing Length, $\mathrm{H}=$ Hubbard, $\mathrm{A}=$ Arbor Acre

The estimate of body weight and body measurements as affected by age and strain of Hubbard and Arbor Acre male strains of broilers chicken are presented in table 1. The result showed that body weight, body length, chest circumference, keel length and wing length were significant $(p<0.05)$ in both strains within the population. There were significant difference in chest circumferenceand wing length between male Hubbard and male Arbor acre at 1weeks of age with higher mean value recorded for male Hubbard, for chest circumference $(10.18 \mathrm{~cm})$ and wing length $(11.00 \mathrm{~cm})$. Also, there were significant difference in body length and keel length at 2 weeks old with male Hubbard broiler had higher mean values for body length $(13.01 \mathrm{~cm})$ and keel length $(6.95 \mathrm{~cm})$. At 3 week of age, there were significant differences in body length, chest circumference and wing length between male Hubbard and male Arbor acre with higher mean value reported for male Hubbard at 3week body length $(15.00 \mathrm{~cm})$, chest circumference $(17.63 \mathrm{~cm})$ and wing length $(18.40 \mathrm{~cm})$. Also at 4 week of age, there were significant differences in body weight and keel length between male Hubbard and male Arbor acre strain of broiler with male Hubbard had higher mean value for body weight $(654.00 \mathrm{~g})$ and keel length $(9.93 \mathrm{~cm})$.

There were significant difference in body weight, chest circumference and keel length between male Hubbard and male Arbor acre strain of broilers with higher mean value reported for male Hubbard strain at 5 week of age, body weight $(862.00 \mathrm{~g})$, chest circumference $(20.93 \mathrm{~cm})$ and keel length $(11.43 \mathrm{~cm})$. There were significant differences in body weight, keel length and wing length at 6 week of age between male Hubbard and male Arbor acre strain of broilers chicken with male Hubbard had 
the higher mean value for body weight $(1133.83 \mathrm{~g})$, keel length $(12.40 \mathrm{~cm})$ and wing length $(25.77 \mathrm{~cm})$. Also, there were significant differences in body weight, body length, keel length and wing length between male Hubbard and male Arbor acre at 7 week of age with male Hubbard reported to had higher mean value for body weight $(1438.50 \mathrm{~g})$, body length $(22.90 \mathrm{~cm})$, keel length $(13.00 \mathrm{~cm})$ and wing length $(27.47 \mathrm{~cm})$. At 8 week of age, there were significant differences in body weight, body length and keel length between male Hubbard and male Arbor acre with male Hubbard strain reported higher mean value for body weight $(1969.70 \mathrm{~g})$, body length $(24.45 \mathrm{~cm})$ and keel length $(13.23 \mathrm{~cm})$.

Table2: Estimate of body weight and body measurement as affected by strain and age of Hubbard and Arbor Acre female strains of broiler chickens

\begin{tabular}{|c|c|c|c|c|c|c|c|c|c|}
\hline \multicolumn{10}{|c|}{ Age } \\
\hline Traits & Strain & 1 & 2 & 3 & 4 & 5 & 6 & 7 & 8 \\
\hline $\begin{array}{l}\text { BDW } \\
(\mathrm{g})\end{array}$ & $\mathrm{H}$ & $\begin{array}{l}112.25 \pm \\
7.25^{\mathrm{a}}\end{array}$ & $\begin{array}{l}218.67 \pm 17.5 \\
2^{\mathrm{a}}\end{array}$ & $\begin{array}{l}341.67 \pm 24 . \\
77\end{array}$ & $\begin{array}{l}449.33 \pm 75.9 \\
3 \mathrm{a}\end{array}$ & $\begin{array}{l}627.67 \pm 65.4 \\
6^{\mathrm{a}}\end{array}$ & $\begin{array}{l}846.33 \pm 27.4 \\
7^{\mathrm{a}}\end{array}$ & $\begin{array}{l}1062.00 \pm 72.1 \\
0^{\mathrm{a}}\end{array}$ & $\begin{array}{l}1326.00 \pm 168.3 \\
5^{\mathrm{a}}\end{array}$ \\
\hline & A & $\begin{array}{l}93.00 \pm 3 . \\
12^{\mathrm{b}}\end{array}$ & $191.33 \pm 8.65$ & $\begin{array}{l}341.00 \pm 25 . \\
51\end{array}$ & $356.67 \pm 9.01^{b}$ & $\begin{array}{l}538.17 \pm 18.6 \\
2^{\mathrm{b}}\end{array}$ & $\begin{array}{l}716.67 \pm 88.1 \\
4^{\mathrm{b}}\end{array}$ & $\begin{array}{l}820.00 \pm 114.1 \\
4^{\mathrm{b}}\end{array}$ & $1013.70 \pm 84.58$ \\
\hline $\begin{array}{l}\mathrm{BDL} \\
(\mathrm{cm})\end{array}$ & $\mathrm{H}$ & $\begin{array}{l}9.38 \pm 0.3 \\
8^{\mathrm{a}}\end{array}$ & $12.93 \pm 0.81^{\mathrm{a}}$ & $13.33 \pm 0.27$ & $14.43 \pm 0.84$ & $17.03 \pm 0.95^{\mathrm{a}}$ & $19.80 \pm 1.20^{\mathrm{a}}$ & $20.33 \pm 0.65^{\mathrm{a}}$ & $21.35 \pm 0.37^{\mathrm{a}}$ \\
\hline & A & $\begin{array}{l}7.77 \pm 0.3 \\
7^{\mathrm{b}}\end{array}$ & $10.60 \pm 0.46^{b}$ & $12.40 \pm 0.21$ & $13.57 \pm 0.68$ & $15.57 \pm 1.23^{b}$ & $15.60 \pm 1.08^{b}$ & $18.67 \pm 2.15^{\mathrm{b}}$ & $19.27 \pm 1.75^{b}$ \\
\hline $\begin{array}{l}\mathrm{CC} \\
(\mathrm{cm})\end{array}$ & $\mathrm{H}$ & $\begin{array}{l}9.73 \pm 0.7 \\
3^{\mathrm{a}}\end{array}$ & $12.78 \pm 0.42^{\mathrm{a}}$ & $15.67 \pm 0.38^{\mathrm{a}}$ & $16.23 \pm 1.49^{\mathrm{a}}$ & $18.47 \pm 0.32^{\mathrm{a}}$ & $20.32 \pm 0.40$ & $22.28 \pm 0.62$ & $24.47 \pm 0.79$ \\
\hline & A & $\begin{array}{l}7.67 \pm 0.4 \\
4^{\mathrm{b}}\end{array}$ & $9.27 \pm 0.24^{b}$ & $13.30 \pm 1.18^{b}$ & $14.38 \pm 0.51^{b}$ & $16.02 \pm 0.67^{b}$ & $19.50 \pm 0.80$ & $21.93 \pm 1.68$ & $23.25 \pm 0.71$ \\
\hline $\begin{array}{l}\mathrm{KL} \\
(\mathrm{cm})\end{array}$ & $\mathrm{H}$ & $\begin{array}{l}3.77 \pm 0.7 \\
3\end{array}$ & $6.38 \pm 0.04^{\mathrm{a}}$ & $7.93 \pm 0.37$ & $9.00 \pm 0.81^{\mathrm{a}}$ & $9.20 \pm 0.17^{\mathrm{a}}$ & $9.97 \pm 0.97$ & $10.45 \pm 1.17$ & $11.43 \pm 0.28$ \\
\hline & A & $\begin{array}{l}2.93 \pm 0.0 \\
3\end{array}$ & $4.53 \pm 0.26^{b}$ & $7.23 \pm 0.97$ & $6.22 \pm 0.50^{b}$ & $7.77 \pm 0.07^{\mathrm{b}}$ & $9.90 \pm 0.36$ & $9.93 \pm 0.09$ & $11.43 \pm 0.28$ \\
\hline $\begin{array}{l}\text { SHL } \\
(\mathrm{cm})\end{array}$ & $\mathrm{H}$ & $\begin{array}{l}2.80 \pm 0.2 \\
3\end{array}$ & $3.67 \pm 0.50$ & $3.87 \pm 0.09$ & $4.23 \pm 0.20$ & $5.30 \pm 0.20$ & $6.20 \pm 0.33^{\mathrm{a}}$ & $6.20 \pm 0.26$ & $6.93 \pm 0.46$ \\
\hline & A & $\begin{array}{l}2.25 \pm 0.2 \\
5\end{array}$ & $3.33 \pm 0.15$ & $3.50 \pm 0.23$ & $3.86 \pm 0.27$ & $4.73 \pm 0.11$ & $4.90 \pm 0.10^{\mathrm{b}}$ & $6.10 \pm 1.10$ & $6.60 \pm 1.25$ \\
\hline $\begin{array}{l}\text { THL } \\
(\mathrm{cm})\end{array}$ & $\mathrm{H}$ & $\begin{array}{l}4.63 \pm 0.6 \\
3\end{array}$ & $6.05 \pm 0.25$ & $6.53 \pm 0.33$ & $8.43 \pm 0.23^{\mathrm{a}}$ & $8.47 \pm 0.17$ & $9.80 \pm 0.58$ & $10.92 \pm 0.57$ & $11.87 \pm 1.13$ \\
\hline & A & $\begin{array}{l}3.43 \pm 0.2 \\
9\end{array}$ & $5.60 \pm 0.60$ & $5.80 \pm 0.15$ & $6.16 \pm 0.11^{b}$ & $7.97 \pm 0.24$ & $8.20 \pm 0.21$ & $9.40 \pm 0.49$ & $10.83 \pm 0.44$ \\
\hline $\begin{array}{l}\text { WL } \\
(\mathrm{cm})\end{array}$ & $\mathrm{H}$ & $\begin{array}{l}10.35 \pm 0 . \\
15\end{array}$ & $12.97 \pm 0.41$ & $16.83 \pm 0.12^{\mathrm{a}}$ & $19.33 \pm 0.09$ & $22.45 \pm 1.56^{\mathrm{a}}$ & $23.80 \pm 1.05$ & $25.40 \pm 0.48$ & $28.42 \pm 0.54$ \\
\hline & A & $\begin{array}{l}9.67 \pm 0.3 \\
8\end{array}$ & $11.90 \pm 1.56$ & $13.70 \pm 0.94^{b}$ & & $19.80 \pm 0.20^{b}$ & $23.33 \pm 0.93$ & $25.23 \pm 1.67$ & $27.10 \pm 1.00$ \\
\hline
\end{tabular}

ab Mean values along the same column with different superscripts are significant at $\mathrm{p}<0.05 \mathrm{BDW}=\mathrm{Body}$ Weight, $\mathrm{BDL}=$ Body Length, $\mathrm{CC}=$ Chest Circumference, $\mathrm{KL}=$ Keel Length, $\mathrm{SHL}=$ Shank Length, THL= Thigh Length, WL= Wing Length, $\mathrm{H}=$ Hubbard, $\mathrm{A}=$ Arbor Acre

Estimate of body weight and body measurements as affected by age and strain of Hubbard and Arbor Acre male strains of broilers are shown in table 2. The result showed that body weight body length, chest circumference, keel length, thigh length and wing length were significant $(p<0.05)$ in both strains within the population. There were significant difference in body weight, body length and chest circumference between female Hubbard and female Arbor acre at 1 weeks of age with higher mean value recorded for male Hubbard, for body weight $(112.25 \mathrm{~g})$, body length $(9.38 \mathrm{~cm})$ and chest circumference $(9.73 \mathrm{~cm})$. Also, there were significant difference in body weight, body length, chest circumference, and keellength at 2 weeks old with Hubbard broiler had higher mean values for body weight $(218.67 \mathrm{~g})$, body length $(12.93 \mathrm{~cm})$, chest circumference $(12.78 \mathrm{~cm})$ and keel length $(6.38 \mathrm{~cm})$ respectively. At 3 week of age, there were significant differences in chest circumference and wing length between male Hubbard and male Arbor acre with higher mean value reported for female Hubbard at 3 week body chest circumference $(15.67 \mathrm{~cm})$ and wing length $(16.83 \mathrm{~cm})$. Also at 8 week, there were significant differences in body weight, chest circumference, keel length and thigh length between male Hubbard and male Arbor acre strain of broiler with male Hubbard had higher mean value for body weight $(449.33 \mathrm{~g})$, chest circumference $(16.23 \mathrm{~cm})$, keel length $(9.00 \mathrm{~cm})$ and thigh length $(8.43 \mathrm{~cm})$. There were significant difference in body weight, body length, chest circumference, keel length and wing length between female Hubbard and female Arbor acre at 5weeks of age with higher mean value recorded for female Hubbard, for body weight $(627.67 \mathrm{~g})$, body length $(17.03 \mathrm{~cm})$, chest circumference $(18.47 \mathrm{~cm})$, keel length $(9.20 \mathrm{~cm})$ and wing length $(22.45 \mathrm{~cm})$. Also, there were significant difference in body weight, body length and shank length at 6weeks old with Hubbard 
broiler had higher mean values for body weight $(846.33 \mathrm{~g})$, body length $(19.80 \mathrm{~cm})$ and shank length $(6.20 \mathrm{~cm})$ respectively. At 7 and 8 week, there were significant differences in body weight and body length between female Hubbard and female Arbor acre with higher mean value reported for female Hubbard at 7 week body weight $(1062.00 \mathrm{~g})$, body length $(20.33 \mathrm{~cm})$ and 8 week body weight $(1326.00 \mathrm{~g})$, body length $(21.35 \mathrm{~cm})$ of age respectively.

Table3. Phenotypic correlation between body weight and body measurements of female and male Arbor Acre strains of broiler

\begin{tabular}{|l|l|l|l|l|l|l|l|}
\hline & BDW $(\mathrm{g})$ & BDL $(\mathrm{cm})$ & CC $(\mathrm{cm})$ & KL $(\mathrm{cm})$ & SHL $(\mathrm{cm})$ & THL $(\mathrm{cm})$ & WL $(\mathrm{cm})$ \\
\hline BDW $(\mathrm{g})$ & & $0.96^{* * *}$ & $0.98^{* * *}$ & $0.86^{* * *}$ & $0.90^{* * *}$ & $0.86^{* * *}$ & $0.95^{* * *}$ \\
\hline BDL $(\mathrm{cm})$ & $0.98^{* * *}$ & & $0.93^{* * *}$ & $0.84^{* * *}$ & $0.90^{* * *}$ & $0.86^{* * *}$ & $0.91^{* * *}$ \\
\hline CC $(\mathrm{cm})$ & $0.97^{* * *}$ & $0.97^{* * *}$ & & $0.92^{* * *}$ & $0.86^{* * *}$ & $0.89^{* * *}$ & $0.96^{* * *}$ \\
\hline KL $(\mathrm{cm})$ & $0.94^{* * *}$ & $0.96^{* * *}$ & $0.96^{* * *}$ & & $0.69^{* * *}$ & $0.90^{* * *}$ & $0.88^{* * *}$ \\
\hline SHL $(\mathrm{cm})$ & $0.95^{* * *}$ & $0.93^{* * *}$ & $0.95^{* * *}$ & $0.91^{* * *}$ & & $0.70^{* * *}$ & $0.83^{* * *}$ \\
\hline THL $(\mathrm{cm})$ & $0.97^{* * *}$ & $0.96^{* * *}$ & $0.96^{* * *}$ & $0.92^{* * *}$ & $0.94^{* * *}$ & & $0.87^{* * *}$ \\
\hline WL $(\mathrm{cm})$ & $0.96^{* * *}$ & $0.97^{* * *}$ & $0.98^{* * *}$ & $0.97 * * *$ & $0.92^{* * *}$ & $0.95^{* * *}$ & \\
& & & & & & & \\
\hline
\end{tabular}

$* * *=$ Very highly significant $(\mathrm{p}<0.0001)$

Upper diagonal $=$ female, Lower diagonal $=$ male, $\mathrm{BDW}=$ Body Weight, $\mathrm{BDL}=$ Body Length, $\mathrm{CC}=$ Chest Circumference, $\mathrm{KL}=$ Keel Length, $\mathrm{SHL}=$ Shank Length, THL= Thigh Length, WL= Wing Length

Phenotypic correlation between body weight and body measurement of female and male Arbor Acre are presented in table 3 . The resulted showed that body weight was positively and significantly $(\mathrm{p}<0.0001)$ correlated with all body measurements in both male and female Arbor Acre within the population. There was statistically significantly $(\mathrm{p}<0.0001)$ positive correlation between body weight and body measurement of female Arbor Acre. The correlation coefficients recorded for female Arbor Acre were body weight/body length $(\mathrm{r}=0.96)$, body weight/chest circumference $(\mathrm{r}=0.98)$, body weight/keel length $(r=0.86)$, body weight/shank length $(r=0.90)$, body weight/thigh length $(r=0.86)$ and body weight/ wing length $(r=0.95)$. Also, there was statistically significantly $(\mathrm{p}<0.0001)$ positive phenotypic correlation between body weight and body measurements in male Arbor Acre. The correlation coefficients recorded for male Arbor Acre strain were body weight/body length $(r=0.98)$, body weight/chest circumference $(\mathrm{r}=0.97)$, body weight/keel length $(\mathrm{r}=0.94)$, body weight $/$ shank length ( $\mathrm{r}=0.95)$, body weight/thigh length $(\mathrm{r}=0.97)$ and body weight/wing length $(\mathrm{r}=0.96)$.

Table4. Phenotypic correlation between body weight and body measurement of female and male Hubbard strain of broiler chicken

\begin{tabular}{|c|c|c|c|c|c|c|c|}
\hline & $\mathrm{BDW}(\mathrm{g})$ & $\mathrm{BDL}(\mathrm{cm})$ & $\mathrm{CC}(\mathrm{cm})$ & $\mathrm{KL}(\mathrm{cm})$ & SHL (cm) & THL (cm) & $\mathrm{WL}(\mathrm{cm})$ \\
\hline $\mathrm{BDW}(\mathrm{g})$ & & $0.88 * * *$ & $0.92 * * *$ & $0.72 * * *$ & 0.90 *** & $0.91 * * *$ & $0.90 * * *$ \\
\hline $\mathrm{BDL}(\mathrm{cm})$ & $0.92 * * *$ & & $0.92 * * *$ & $0.81 * * *$ & 0.90 *** & $0.89 * * *$ & $0.90 * * *$ \\
\hline $\mathrm{CC}(\mathrm{cm})$ & $0.93 * * *$ & $0.95 * * *$ & & $0.88 * * *$ & $0.93 * * *$ & $0.91 * * *$ & $0.88 * * *$ \\
\hline $\mathrm{KL}(\mathrm{cm})$ & $0.72 * * *$ & $0.82 * * *$ & $0.88 * * *$ & & $0.81 * * *$ & $0.82 * * *$ & $0.71 * * *$ \\
\hline SHL $(\mathrm{cm})$ & $0.93 * * *$ & $0.94 * * *$ & $0.90 * * *$ & $0.77 * * *$ & & $0.93 * * *$ & $0.89 * * *$ \\
\hline THL (cm) & $0.84 * * *$ & $0.92 * * *$ & $0.89 * * *$ & $0.78 * * *$ & $0.92 * * *$ & & $0.90 * * *$ \\
\hline $\mathrm{WL}(\mathrm{cm})$ & $0.85^{* * *} *$ & $0.88 * * *$ & $0.83 * * *$ & $0.66 * * *$ & $0.92 * * *$ & $0.90 * * *$ & \\
\hline
\end{tabular}

*** Very highly significant $(\mathrm{p}<0.0001)$

Upper diagonal $=$ female, Lower diagonal $=$ male, $\mathrm{BDW}=$ Body Weight, $\mathrm{BDL}=$ Body Length, $\mathrm{CC}=$ Chest Circumference, $\mathrm{KL}=$ Keel Length, $\mathrm{SHL}=$ Shank Length, THL= Thigh Length, WL= Wing Length

Phenotypic correlations between body weight and body measurements of both female and male Hubbard strain are presented in table 4 . The result obtained revealed that body weight and body measurements were positively and significantly $(\mathrm{p}<0.0001)$ correlated in both female and male Hubbard within the population. The correlation coefficient for female Hubbard were body weight/body length $(\mathrm{r}=0.88)$, body weight/chest circumference $(\mathrm{r}=0.92)$, body weight/keel length $(\mathrm{r}=0.72)$, body weight/shank length $(\mathrm{r}=0.90)$, body weight/thigh length $(\mathrm{r}=0.91)$ and body weight/wing length $(\mathrm{r}=0.90)$. Also, the correlation coefficient for male Hubbard were body weight/body length $(\mathrm{r}=0.92)$, body weight/chest circumference $(\mathrm{r}=0.93)$, body weight/keel length $(\mathrm{r}=0.72)$, body weight/shank length $(\mathrm{r}=0.93)$, body weight/thigh length $(\mathrm{r}=0.84)$ and body weight/wing length $(\mathrm{r}=0.85)$. 


\section{DISCUSSION}

The results on estimate of body weight and body measurements revealed that both male and female Hubbard strain had the higher mean value of body weight and body measurements and this could be due to differences in genetic makeup of the birds. These results confirm the fact that genotype sets a ceiling on the body weight capacity since in the present study, nutrition and other environmental factors were uniform. This agrees with the statement of Biligih (1995) that body weight is influenced not only by genetic makeup but also by environmental factors. Hubbard broiler strains exhibited consistent superior body weight difference over the Arbor acre strain at each age group, a trend that was similarly reflected with the body measurements and this agrees with the findings of Yahaya et al. (2012) who reported that the linear body measurements of Hubbard strain were significantly higher than that of Arbor Acre strain at 8 weeks of age for all body parameter studied, but the value obtained in this present study were lighter than those for Hubbard and Arbor acre strains obtained by Yahaya $e t$ al. (2012) at 2,4, 6 and 8 weeks of age. The relatively lighter means body weight and body measurements obtained in this study could be due to differences in feed composition, environmental condition or management system.

The results on phenotypic correlation showed that body weight of both Arbor Acre and Hubbard strains were positively and significantly phenotypic correlated with all body measurements within the population. There were significantly and positive phenotypic correlation between body weight and body measurements in Arbor Acre and Hubbard strains of broiler chicken. This means there was strong association between body weight and body measurement. Ajayi et al. (2008) reported that an increase in any of the body measurement will invariably lead to corresponding increase in body weight of chicken. Body weight related highly and positively with all the original body measurements of the chickens. The high positive correlation values indicative that as live body weight increases, the linear body measurements will also increase (Egena, 2014). Also, there were significantly and positive phenotypic correlation between body weight and body measurements in Hubbard strain of broiler chicken. The result implies that linear body measurements are good indicators of body weight. This was in agreement with the observations of previous researchers (Ojedapo et al., 2006; Ige et al., 2007). The body weight was positively and significantly phenotypic correlated with all body measurements in both male and female Arbor Acre within the population. Simeon, (2014) reported that there was statistically significant positive phenotypic correlation between body weight and linear body measurements in Dominant Black pullets. Ojedapo et al. (2006) reported similar results in three strains of commercial layers. The result obtained also revealed that body weight and body measurements were positively and significantly phenotypic correlated in both female and male Hubbard within the population. This implies that there exist a strong linear relationship between body weight and body length, chest circumference, keel length, shank length, thigh length and wing length. This result suggests that an improvement in body weight of commercial broiler chickens would lead to an improvement in linear body parameters. This result also corroborates the reports of Yahaya et al., (2012) and Alabi et al. (2012) that high positive correlation exist between body weight and linear body measurements in broilers and naked neck/venda chickens of South Africa, respectively. A similar result was reported by Momoh and Kershima (2008) using Nigerian local chickens.

\section{CONCluSion}

From this study, Hubbard broiler strain has been seen to exhibit significant higher body weight and body measurements over Abor acre strain of broiler. It also suggests that there are high positive correlations between body weight and linear body measurements. The implication is that an improvement in body measurements could lead to an improvement in body weight. This relationship may be used in selection programme for further genetic improvement of commercial broiler chickens.

\section{REFERENCES}

[1] Ajayi, F. O. and EjioforO., 2009.Effect of genotype $\mathrm{x}$ sex interaction on growth and some developmentcharacteristics of Ross and Anak broiler strains inthe high rainforest zone of Nigeria.Asian J.Poult. Sci. 3(2), $51-56$.

[2] Ajayi, F. O. Ejiofor, O. Ironke, M. O., 2008. Estimation of body weight from linear body measurements in two commercial meat-type chickens. Global J. Agric. Sci.7 (1),57-59.

[3] Alabi, O.J. Ng'ambi, J.W., Norris, D. and Egena, S.S.A., 2012. Comparative Study of Three Indigenous Chicken Breeds of South Africa: body Weight and Linear body measurements. Agri. J. 7(3), $220-225$. 
[4] Bilgili, S.F, and Hess, J.B., 1995. Placement density influences broiler carcass grade and meat yields. J.Appl. Poult. Res. 4:384-389.

[5] Cahaner, A. 2008.Breeding broilers for hot conditions.Nigerian Poult. Sci. J. 5, 156-163.

[6] Egena S. S. A., Ijaiya, A. T., Ogah, D. M. and aya, V. E. 2014.Principal Component Analysis of Body Measurements in A Population of Indigenous Nigerian Chickens Raised Under Extensive Management System. Slovak J. Ani. Sci. 47(2),77-82.

[7] Emmerson, D. A. 1997. Commercial approaches to genetic selection for growth and feed conversion in domestic poultry. Poult. Sci. 76 (8), 1121-1125.

[8] FAO. 2006a. World agriculture: towards 2030/2050 interim report. Rome.

[9] Ige, A. O., Salako, A. E., Ojedapo, L. O., Adedeji, T. A., Yakubu, A., Amao, S.R., Animasahun, A. O. and Amao,O. A. 2007 Prediction of body weight on the basis of body measurements in mature indigenous chickens in derived savannah zone of Nigeria. In: Proceedings of the 32nd annual conference, Nigeria Society for Animal Production, 18-21 March, 2007, Calabar, Nigeria. Pp185-187.

[10] Ige, A.O. 2013 Estimation of Genetic Parameter in Yoruba and Fulani Ecotypes Indigenous Chickens of Nigeria. Transnational J. Sci. and Tech. 3(10), 1-28.

[11] Jabbar, M.A. 2004. Smallholder livestock for poverty alleviation: Issues and approaches, Keynote paper presented at Bangladesh Agril. Res. Coun., 12 May, Dhaka, Bangladesh

[12] Jaturasitha S. 2004. Meat management. Mingmuang Press, Chiang Mai, Thailand.

[13] Momoh, O.M. and Kershima, D.E. 2008 Linear body measurements as predictors of body weight in Nigerian local chickens. Asset series A 8(2):206 - 212.

[14] Obasoyo, D. O., Bamgbose, A. M and Omoikhoje, S. O. 2005. Blood profile of broilers fed diets containing different animal protein feedstuff. $10^{\text {th }}$ Annual Conf. Anim. Sci. Assocition of Nigeria.Univ of Ado- Ekiti, Nigeria.176 - 178.

[15] Oguntoyinbo,J.S.,1978.Vital Statistics in Ogbomoso Cofile:///C:/Users/Lisa\%20Gabrielson/

Downloads/135140\%20Vol.\%202,\%20Issue\%204\%20201.pdfmmunity.1st Edn., Daybis Publishing Ltd., Ibadan, Nigeria.

[16] Oluyemi, J.A. and Roberts, F.A., 2000.Poultry production in warm wet climates. 2nd Edn., Spectrum Books Ltd., Ibadan, 244 pp.

[17] Razuki, W. M., Mukhlis, S. R., Jasim, F. H and Hamad, R. F. 2011. Productive performance of four broiler genotypes reared under high ambient temperature. Inter. J. Poult. Sci. 10(2), 87-92.

[18] Simeon, O. O. 2014. Interrelationships and Phenotypic Correlations among Body Dimensions in Commercial Pullets Reared in the Derived Savannah Zone of Nigeria. International Journal of Agriculture, Forestry and Fisheries. 2(2), 35-39.

[19] Yahaya, H.K. Brahim, H. and Abdul Salam, S 2012.Comparative Study of the Body Weight and Body Conformations of Two Broiler Strains under the Same Dietary Condition.Inter. J. of Ani.and Vet. Advances 4(3), 195-197. 